\title{
CARACTERIZAÇÃO DAS VIBRAÇÕES MECÂNICAS NO E RUÍDO NO POSTO DE OPERAÇAO NO TRATOR AGRÍCOLA MODIFICADO "TRANSFORMAX"
}

\author{
Paulo Roberto Forastiere ${ }^{1}$, Haroldo Carlos Fernanades ${ }^{2}$, Mauri Martins Teixeira ${ }^{3}$, Paulo Roberto Cecon ${ }^{4}$, Diego \\ Weslley Ferreira do Nascimento Santos ${ }^{5}$
}

\begin{abstract}
RESUMO
Os estudos ergonômicos visam adaptar o trabalho ao homem por meio da análise da tarefa, da postura e dos movimentos do trabalhador, assim como de suas exigências físicas e psicológicas, no intuito de reduzir a fadiga e o estresse, proporcionando um posto de trabalho confortável e seguro. Um dos fatores indesejáveis que ocorre em tratores agrícolas é a vibração, não só por causa do desconforto produzido e possível degradação da saúde do operador, mas também por causa do aumento das tensões dinâmicas que podem levar à fadiga e insuficiência dos elementos do trator. Já o ruído excessivo pode causar diminuição ou perda da capacidade auditiva do operador quando o tempo de exposição for muito longo. Dessa forma, objetivou-se, avaliar os níveis de ruído e vibração no posto de operação do trator agrícola modificado Transformax ${ }^{\circledR}$, em função da força de tração e rotação do eixo motriz, com tipos de rodados, com largura de 13.6 e 18.4. Para esse experimento, foi realizada uma análise de espectro de frequências para todos os tratamentos com intuito de verificar o comportamento das vibrações verticais e longitudinais na base do posto de operação, de acordo com cada faixa de frequência e os níveis de ruído foram determinados com auxílio de um decibelímetro digital, posicionado próximo ao ouvido do operador. As variáveis, força de tração e rotação do eixo motriz, não apresentaram efeito significativo nos níveis de ruído no posto de operação do trator, entretanto, os níveis médio de ruído foram mais elevados no trator quando equipado com pneu 18.4.
\end{abstract}

Palavras-chave: ergonomia, espectro de frequência, tratores

\section{ASBTRACT \\ VIBRATIONS OF CHARACTERIZATION IN MECHANICAL AND NOISE IN OPERATION DESK IN THE AGRICULTURAL TRACTOR MODIFIED "TRANSFORMAX"}

The ergonomic studies aimed at adapting the work to man, through the task analysis, posture and worker movements, as well as their physical and psychological requirements, in order to reduce fatigue and stress, providing a comfortable job and safe. One of the undesirable factors occurring in agricultural tractors is vibration, not only because the produced discomfort, and possible degradation of the operator's health, but also because of the increased dynamic stresses that can lead to fatigue and failure of the tractor elements. Already excessive noise can cause decrease or loss of hearing ability when the exposure time is too long. Thus, this study aims to assess the levels of noise and vibration in the agricultural tractor operator station Transformax ${ }^{\circledR}$ modified, depending on the traction force and rotation of the drive shaft, with types of rotated, of width 13.6 and 18.4. For this experiment was performed a frequency spectrum analysis for all treatments in order to verify the behavior of the vertical and longitudinal vibrations at the base of the operator station, according to each frequency band and the noise levels were determined with the aid of a digital noise, positioned near the operator's ear. The variables, traction force and rotation of the drive shaft showed no significant effect on noise levels in tractor operator station, however, the average noise levels were higher in the tractor equipped with tires 18.4.

Keywords: ergonomics, frequency spectrum, tractors

Recebido para publicação em 20/04/2016. Aprovado em 08/06/2016.

1 - Engenheiro Agrônomo, doutorando da UFV/Viçosa-MG, paulo.forastiere@gmail.com

2 - Engenheiro Agrícola e Ambiental, Prof. Doutor da UFV/Viçosa-MG, haroldouvf@gmail.com

3 - Engenheiro Agrônomo, Prof. Doutor da UFV/Viçosa-MG, mauriufv@gmail.com

4 - Engenheiro Agrônomo, Prof. Doutor da UFV/Viçosa-MG, cecon@ufv.br

5 - Engenheiro Florestal, doutorando da UFV/Viçosa-MG, diegoweslley89@hotmail.com 


\section{INTRODUÇÃO}

Atendendo a tendência de ocupação de grandes áreas e perspectivas de grande expansão do cultivo de importantes produtos agrícolas, a utilização de máquinas agrícolas é de extrema importância (SILVA et al. 2011). Assim, o processo de mecanização exige que o operador esteja inteiramente adaptado ao posto de trabalho para poder exercer sua função.

Desta forma, a ergonomia é definida como a adaptação do trabalho ao homem. Sendo que o trabalho abrange as máquinas, os equipamentos e também toda a situação em que ocorre o relacionamento entre o homem e seu trabalho (LIDA, 2003; FERNANDES et al., 2010). Os estudos ergonômicos visam adaptar o trabalho ao homem através da análise da tarefa, da postura e dos movimentos do trabalhador, assim como de suas exigências físicas e psicológicas, a fim de reduzir a fadiga e o estresse, proporcionando um posto de trabalho confortável e seguro. Com isso, reduzindo o cansaço mental e físico dos operadores e, consequentemente, aumentando a eficiência no trabalho (KILESSE, et. al., 2006).

Segundo Dhingra et al. (2003), a palavra conforto pode ser "uma harmonia agradável entre fatores fisiológicos, psicológicos e físicos, entre um ser humano e o ambiente" ou, ainda, a "ausência de desconforto". Um local de trabalho deve ser sadio e agradável, proporcionando o máximo de proteção, sendo o resultado de fatores materiais ou subjetivos, que previne acidentes, doenças ocupacionais, além de proporcionar melhor relacionamento entre a empresa e o empregado.

Além disso, o desconforto extremo acaba por diminuir o rendimento e aumenta a fadiga, o que leva, muitas vezes, o trabalhador ao estresse. Diversos trabalhadores executam suas atividades em ambientes inadequados, expostos a elevados níveis de ruído e vibração, iluminação deficiente e mal distribuída, condições climáticas desfavoráveis (temperatura, umidade relativa, direção e intensidade do vento) e elevados níveis de gases de exaustão, fuligens e poeiras. Esses fatores influenciam diretamente o desempenho, a segurança e a saúde dos mesmos (FIEDLER et al. 2010).

Um dos fatores indesejáveis que ocorre em tratores agrícolas é a vibração, não só por causa do desconforto produzido, e possível degradação da saúde do operador, mas também por causa do aumento das tensões dinâmicas que podem levar à fadiga e insuficiência dos elementos do trator (METHA et al. 2000, LOUTRIDIS et al., 2011).

De acordo com Kroemer e Grandjean (2005), as vibrações são oscilações da massa em função de um ponto fixo, produzidas por movimentos periódicos regulares ou irregulares de uma ferramenta, veículo ou outro mecanismo em contato com o corpo humano, deslocando-o da sua posição de repouso.

Normalmente, as vibrações são reduzidas com a utilização de uma suspensão de amortecimento de vibração no assento. Particularmente, para tratores agrícolas, soluções integradas que são capazes de reduzir as vibrações transmitidas ao operador podem ser adotadas, como sistemas de suspensão com molas e amortecedores para os eixos dianteiros e traseiros e suspensão no posto de operação de tratores agrícolas (MARSILI et al., 2002; BOAUZARA et al., 2006; SCARLETT et al., 2007).

Já o ruído excessivo pode causar diminuição ou perda da capacidade auditiva quando o tempo de exposição for muito longo. A Associação Brasileira de Normas Técnicas (ABNT) estabelece os níveis de ruído para conforto acústico, sendo o nível de ruído de $85 \mathrm{~dB}$ permitido por 8 horas de exposição (BRASIL, 2014).

Partindo da necessidade de atender as normas técnicas, objetivou-se com esse trabalho avaliar os níveis de ruído e vibração no posto de operação do trator agrícola modificado Transformax ${ }^{\circledR}$, em função da força de tração e rotação do eixo motriz, com tipos de rodados, 13.6 e 18.4 .

\section{MATERIAL E MÉTODOS}

Os experimentos foram realizados na fazenda São Domingos, no município de Engenheiro Beltrão

\section{REVENG}




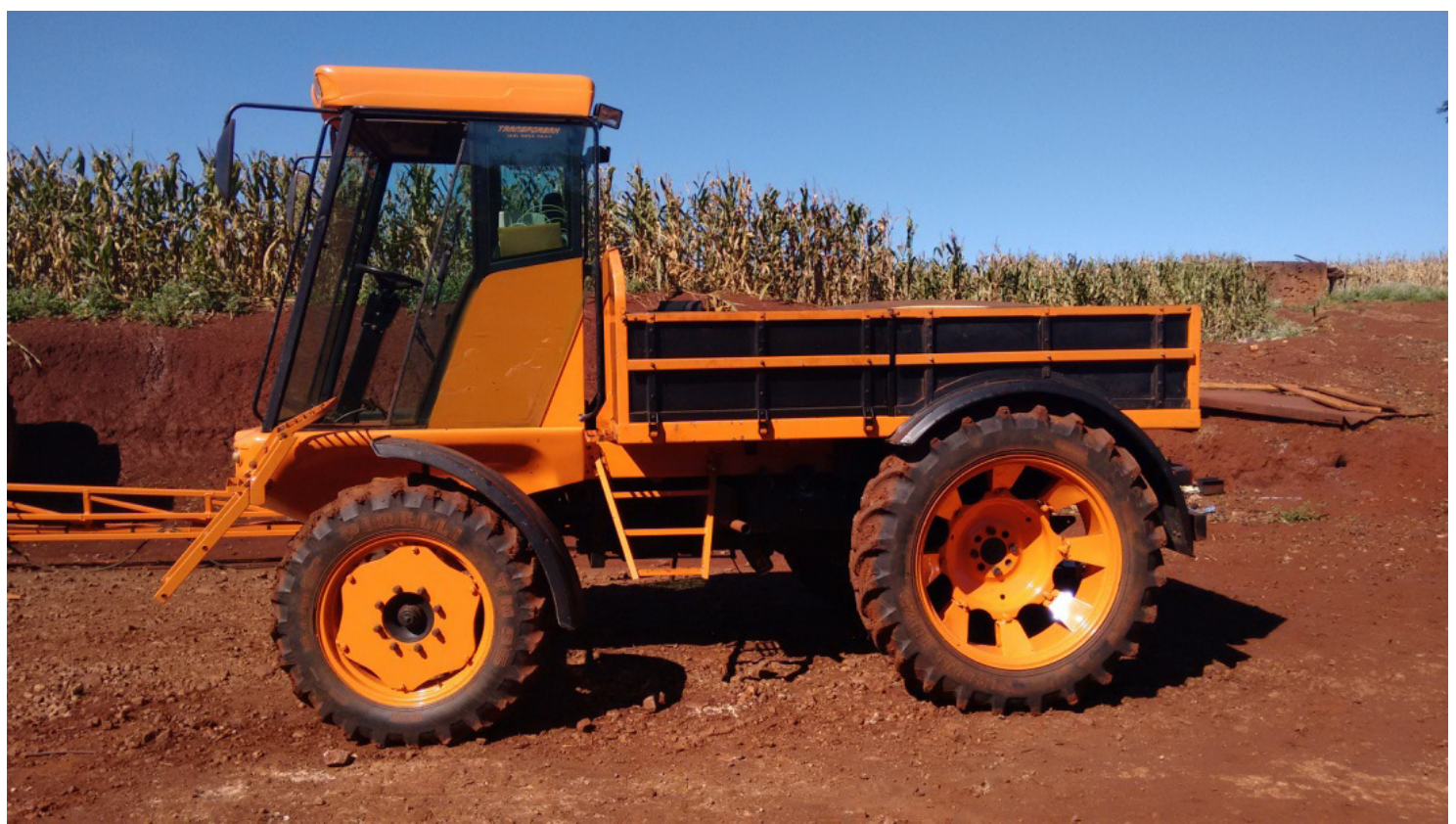

Figura 1. Transformax ${ }^{\circledR}$ configuração trator agrícola.

- PR, com latitude $23^{\circ} 50^{\prime} 53^{\prime \prime}$ Sul e longitude $52^{\circ}$ 19' 27' Oeste segundo datum horizontal WGS84. O trabalho teve como alvo um trator agrícola modificado denominado Transformax ${ }^{\circledR}$, construído a partir do chassi monobloco de um trator FORD, modelo $66004 \times 2$, com 56,6 kW (77 cv) de potência nominal no motor (Figura 1). Durante os testes a máquina foi analisada na configuração trator agrícola.

As avaliações foram realizadas em uma pista de solo compactado, com 40 metros de comprimento e 4 metros de largura, com declividade inferior a $1 \%$. O solo da região segundo EMBRAPA (2006) é classificado como Nitossolo Vermelho Eutroférrico.

O experimento foirealizado em um delineamento inteiramente casualizado, disposto em um esquema fatorial $3 \times 4$, sendo três rotações do eixo traseiro $(14,33 ; 23,92$ e $32,62 \mathrm{rpm})$, e quatro forças na barra de tração, totalizando 48 repetições. A força na barra de tração foram fornecidas por um trator carga, monitoradas com o auxílio de uma célula de carga, marca $\mathrm{Kratos}^{\circledR}$, com capacidade para 50 $\mathrm{kN}$ e com sinal de resposta 306,63 $\mathrm{N} \mathrm{Mv}^{-1}$ ligada ao sistema de aquisição de dados (Quadro 1), analisando a influência da velocidade de trabalho e da força de tração desenvolvida pelo espécime em função de dois modelos de pneus (Pirelli ${ }^{\circledR}$ TM
75 13.6-38 e Pirelli ${ }^{\circledR}$ TM 95 18.4-34), as pressões de insuflação dos pneus foram $241,31 \mathrm{kPa}$ (35 psi) para o modelo $13.6-38$ e $137,89 \mathrm{kPa}$ (20 psi) para o modelo $18.4-34$, proporcionando o raio de rolamento de 0,8241 e $0,8573 \mathrm{~m}$ respectivamente, e a razão de redução de percurso foi obtida da relação entre velocidade translacional e rotacional para cada um dos rodados da máquina, conforme a Equação 1.

$P_{t}=\frac{V_{r}-V_{t}}{V_{r}}$

em que,

$\mathrm{P}_{\mathrm{t}}=$ ração de redução de percurso (\%);

$\mathrm{V}_{\mathrm{r}}=$ velocidade rotacional $\left(\mathrm{m} \mathrm{s}^{-1}\right) ; \mathrm{e}$,

$\mathrm{V}_{\mathrm{t}}^{\mathrm{r}}=$ velocidade translacional $\left(\mathrm{m} \mathrm{s}^{-1}\right)$.

As vibrações no posto de operação foram obtidas por dois acelerômetros uniaxiais da marca PCB $^{\circledR}$, com faixa de operação de 1 a $4000 \mathrm{~Hz}$ com sensibilidade de $101,5 \mathrm{mV} \mathrm{g}^{-1}$ para o horizontal e $102,8 \mathrm{mV} \mathrm{g}^{-1}$ para o vertical. Os acelerômetros foram instalados perpendiculares entre si, embaixo do acento do operador, representados pelo valor da aceleração média quadrática (Root Mean Square RMS). 
Quadro 1. Marchas utilizadas no trator lastro com suas respectivas forças de tração padronizadas

\begin{tabular}{|c|c|c|c|c|}
\hline Pneu & Força & Marcha & Força padrão (kN) & CV (\%) \\
\hline \multirow{4}{*}{$18.4-34$} & 1 & $4^{\mathrm{a}}$ Simples & 5,10 & 6,73 \\
\hline & 2 & 1․ Simples & 10,54 & 13,62 \\
\hline & 3 & 4a Reduzida & 14,29 & 17,14 \\
\hline & 4 & 3a Reduzida & 18,57 & 12,99 \\
\hline Pneu & Força & Marcha & Força padrão (kN) & CV (\%) \\
\hline \multirow{4}{*}{$13.6-38$} & 1 & 4a Simples & 5,64 & 3,50 \\
\hline & 2 & 1 Simples & 9,76 & 10,61 \\
\hline & 3 & 4a Reduzida & 13,65 & 15,66 \\
\hline & 4 & 3ㄹ Reduzida & 18,64 & 13,12 \\
\hline
\end{tabular}

Foi realizada uma análise de espectro de frequências para todos os tratamentos com intuito de verificar o comportamento das vibrações verticais e longitudinais na base do posto de operação, de acordo com cada faixa de frequência. Os espectros de frequências foram obtidos mediante a conversão dos dados de vibração instantânea do domínio do tempo para o domínio da frequência, com a utilização da transformada rápida de Fourier (TRF). A amplitude de vibração foi calculada com base na raiz da soma quadrática dos coeficientes obtidos na série trigonométrica de Fourier, que tomou um número de $2^{10}$ dados consecutivos de vibração para cada tratamento experimental. Esses resultados foram expressos em forma de gráficos contendo as frequências no eixo das abscissas e a amplitude em termos de vibração RMS no eixo das ordenadas.

Os níveis de ruído foram determinados com auxílio de um decibelímetro digital da marca MINIPA, modelo MSL - 1350, posicionado próximo ao ouvido do operador, operando no circuito de compensação "A", o qual origina níveis de pressão sonora ponderadas em "A". Foram coletados os níveis médios de ruído ao longo das parcelas com escala para leitura em decibel (dB).

Os dados foram avaliados por análise de regressão e os modelos escolhidos com base na significância dos coeficientes de regressão, utilizando-se o teste $\mathrm{t}$, ao nível de $5 \%$ de probabilidade. Para a realização dos procedimentos estatísticos foi utilizado o programa computacional R (R DEVELOPMENT CORE TEAM, 2012).

\section{RESULTADOS E DISCUSSÃO}

As variáveis independentes apresentaram estatisticamente efeito linear significativo e positivo na aceleração longitudinal no posto de operação do trator avaliado (Figura 2).

Para uma mesma força de tração o incremento de uma unidade na rotação do eixo motriz correspondeu a um acréscimo de $0,0106 \mathrm{~m} \mathrm{~s}^{-2}$ na aceleração longitudinal no posto de trabalho da máquina avaliada. $\mathrm{O}$ acréscimo de uma unidade na força de tração para uma mesma rotação do eixo motriz apresentou um aumento de $0,0152 \mathrm{~m} \mathrm{~s}^{-2}$ na aceleração longitudinal no posto de operação do trator.

A variável independente rotação do eixo motriz apresentou efeito linear positivo, porém não significativo ao nível de $5 \%$ de probabilidade pelo teste $t$, na vibração vertical no posto de operação do trator avaliado. Entretanto, a variável força de tração apresentou efeito linear e positivo na aceleração na cabine. Com o aumento de uma unidade na força de tração para uma mesma rotação do eixo motriz houve um incremento de $0,0452 \mathrm{~m}$ $\mathrm{s}^{-2}$ na aceleração vertical na cabine. $\mathrm{O}$ acréscimo de uma unidade na rotação do eixo motriz para uma mesma força de tração apresentou um aumento de $0,0010 \mathrm{~m} \mathrm{~s}^{-2}$ na aceleração vertical no posto de operação do trator avaliado equipado com pneu 13.6.

O trator equipado com rodado 13.6 operando na faixa de força $5,10 \mathrm{kN}$ e rotação do eixo $14,33 \mathrm{rpm}$ apresentou valor de aceleração vertical superior a longitudinal, em torno de $9 \%$. Na condição da força de tração $18,64 \mathrm{kN}$ e rotação do eixo motriz $32,62 \mathrm{rpm}$ a aceleração vertical atingiu $0,87 \mathrm{~m} \mathrm{~s}^{-2}$, enquanto que a aceleração longitudinal apresentou um valor de $0,62 \mathrm{~m} \mathrm{~s}^{-2}$. Ao passar da menor unidade de força e rotação do eixo, para maior força e 


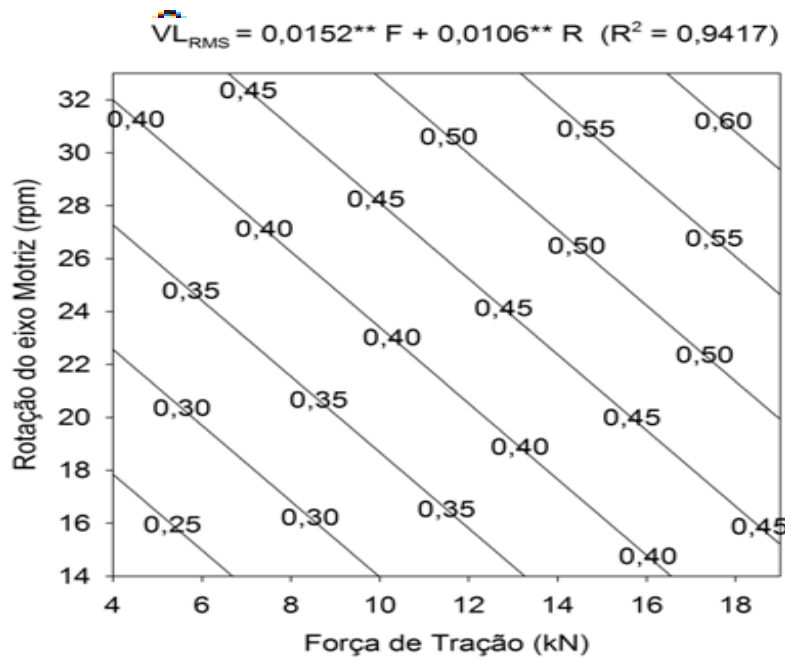

(a)

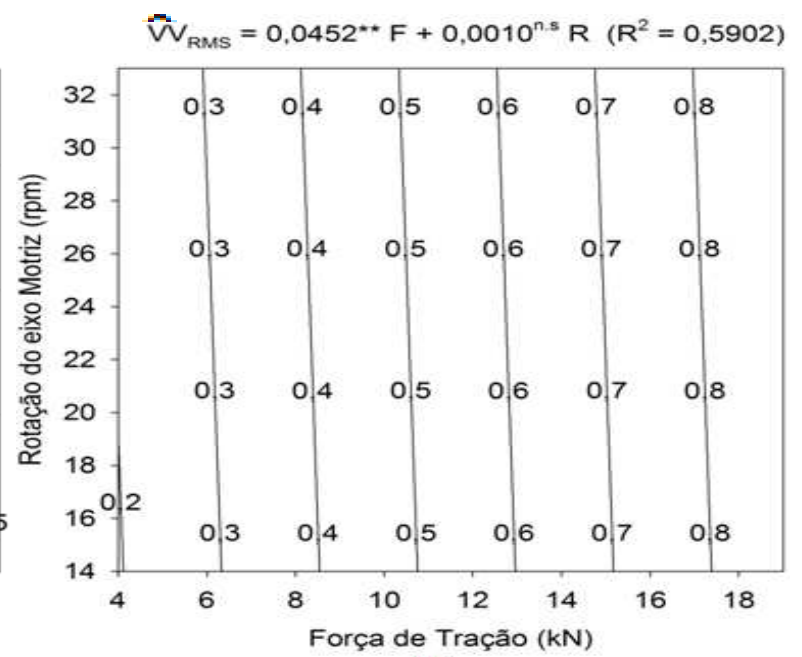

(b)

Figura 2. Vibração Longitudinal $\left(\mathrm{VL}_{\mathrm{RMS}}\right)$ (a) e Vibração Vertical $\left(\mathrm{VV}_{\mathrm{RMS}}\right)$ (b) no posto de operação do trator avaliado equipado com pneu 13.6 em função da força de traça $(\mathrm{F}-\mathrm{kN})$ e rotação do eixo motriz ( $\mathrm{R}$ - rpm), equação ajustada e o coeficiente de determinação $\left(\mathrm{R}^{2}\right) * *$ - Significativo ao nível de 5\% de probabilidade pelo teste $t \mathrm{e}^{\text {n.s }}$ - não significativo ao nível de $5 \%$ de probabilidade.

rotação do eixo motriz, tem-se um acréscimo de $0,40 \mathrm{~m} \mathrm{~s}^{-2}$ para aceleração longitudinal e para vertical $0,63 \mathrm{~m} \mathrm{~s}^{-2}$.

Provavelmente este comportamento superior da aceleração vertical esta relacionado com a característica construtiva do pneu avaliado, por apresentar uma reduzida banda de rodagem, que o torna mais rígido, transmitindo o impacto proveniente do contato com a pista para o sistema de suspensão adicionado à máquina, composto por molas e amortecedores que trabalham em sentido vertical (para cima e para baixo), contribuindo para o valor superior da vibração vertical no posto de operação do trator.

Os níveis de vibração vertical na base do posto de operação foram mais pronunciados com o incremento da força de tração, que está relacionada com os índices de patinagem apresentado pelo trator equipado com o pneu 13.6. O deslizamento é fonte de instabilidade vertical, contribuindo assim com o acréscimo das acelerações verticais.

Avaliando a aceleração no posto de operação de um trator $4 \times 2$ TDA cabinado, com potência no motor de $63 \mathrm{~kW}$ em função da velocidade de deslocamento e profundidade de trabalho de um escarificador, Pinho et al. (2014) encontraram que a velocidade de $4,5 \mathrm{~km} \mathrm{~h}^{-1}$ proporcionou um aumento de $13,5 \%$ maior de acelerações do que a velocidade de $3,5 \mathrm{~km} \mathrm{~h}^{-1}$, na direção vertical, que de acordo com a norma (ISO 2631-1, 1997) estão classificadas como máquinas extremamente desconfortáveis, cujo limite é de $2,0 \mathrm{~m} \mathrm{~s}^{-2}$.

A variável força de tração apresentou efeito linear positivo na aceleração longitudinal no posto de trabalho do trator avaliado, entretanto não houve diferença significativa (Figura 3(a)). No entanto, para uma mesma rotação do eixo motriz, o incremento de uma unidade da força de tração resultou num acréscimo dos níveis de vibração horizontal em $0,0036 \mathrm{~m} \mathrm{~s}^{-2}$.

A variável rotação do eixo motriz apresentou efeito linear positivo e significativo na vibração longitudinal no posto de operação do trator avaliado. Com o acréscimo de uma unidade na rotação do eixo motriz para uma mesma força de tração, correspondeu a um incremento de $0,0115 \mathrm{~m}$ $\mathrm{s}^{-2}$ na vibração longitudinal.

O mesmo comportamento não pode ser observado na vibração vertical (Figura 3(b)). As variáveis força de tração e rotação do eixo motriz apresentaram efeito linear positivo e significativo na vibração vertical no posto de operação do trator avaliado. Com o incremento de uma unidade na força de tração para uma mesma rotação do eixo motriz ocasionou um acréscimo de $0,0105 \mathrm{~m} \mathrm{~s}^{-2}$ no nível de vibração vertical no posto de trabalho da máquina. De modo equivalente, para uma mesma força de tração, o aumento de uma unidade na rotação do eixo motriz resultou no acréscimo 


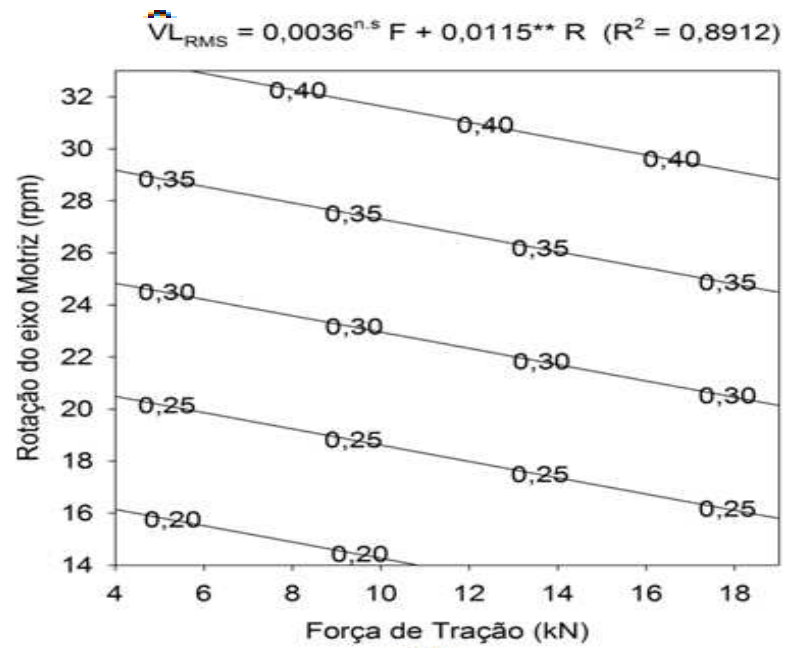

(a)

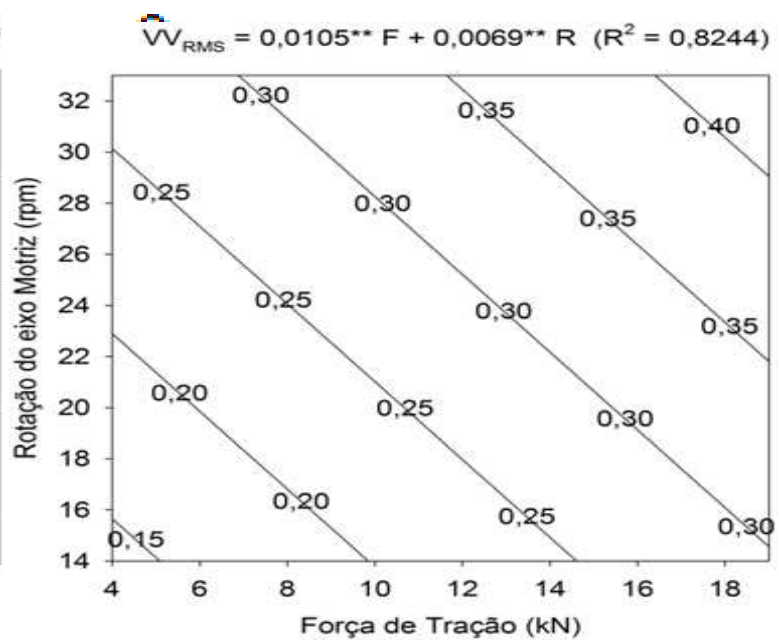

(b)

Figura 3. Vibração Longitudinal $\left(\mathrm{VL}_{\mathrm{RMS}}\right)(\mathrm{a})$ e Vibração Vertical $\left(\mathrm{VV}_{\mathrm{RMS}}\right)$ (b) no posto de operação do trator avaliado equipado com pneu 18.3 em função da força de traça $(\mathrm{F}-\mathrm{kN})$ e rotação do eixo motriz ( $\mathrm{R}$ - rpm), equação ajustada e o coeficiente de determinação $\left(\mathrm{R}^{2}\right)^{* *}$ - Significativo ao nível de $5 \%$ de probabilidade pelo teste $t \mathrm{e}^{\text {n.s }}$ - não significativo ao nível de $5 \%$ de probabilidade.

$0,0069 \mathrm{~m} \mathrm{~s}^{-2}$ na vibração vertical no posto de operação do trator avaliado.

Operando na menor rotação do eixo motriz $(14,33)$ e tracionando a menor carga aplicada na barra de tração $(5,64 \mathrm{kN})$, a aceleração longitudinal foi $20 \%$ superior a aceleração vertical no posto de operação da máquina. Na maior condição de força $(18,64 \mathrm{kN})$ e rotação $(32,62 \mathrm{rpm})$ que o trator foi submetido a aceleração longitudinal foi superior a aceleração vertical apenas $4,7 \%$. Este fato pode ser explicado pela eficiência da união do conjunto, suspensão constituída de molas e amortecedores e das características construtivas do pneu 18.4, que contribuíram em absorver o impacto do rodado com a pista de rolamento, reduzindo a magnitude da vibração vertical no posto de operação do trator avaliado.

A variável rotação do eixo motriz apresentou maior influência na vibração vertical no posto de operação do trator avaliado, entretanto este comportamento pode ser justificado pelo pneu 18.4 apresentar menor patinagem, contribuindo para maior velocidade de deslocamento, consequentemente, proporcionando maior instabilidade ao trator, demonstrando ineficiência da suspensão da máquina em minimizar a aceleração longitudinal no posto de operação.

Cunha et al. (2009), ao estimarem a vibração no posto de operação de um trator $4 \times 2$ com potência nominal no motor de $60,35 \mathrm{~kW}$ equipado com pneu 18.4, tralhando com arado de disco e uma grade, encontraram maiores valores de vibração na direção de deslocamento do trator, ultrapassando os limites aceitáveis pela ISO 2631-1 (1997), podendo trazer danos a espinha dorsal dos operadores de máquinas agrícolas. Resultados semelhantes foram encontrados por Ribas et al. (2014) que, em estudos da exposição humana à vibração de corpo inteiro em um trator agrícola de $75 \mathrm{~kW}$ na operação de semeadura, encontraram maiores valores de vibração longitudinal, ou seja, para uma jornada de 8 horas de trabalho, o operador está exposto acima do nível limite de conforto estabelecido pela norma utilizada.

Relacionando a vibração longitudinal no posto de operação, Langer et al. (2015) avaliaram um trator agrícola com $120 \mathrm{~kW}$ de potência no motor com e sem acionamento da TDA, tracionando uma enfardadora, e verificaram que o acionamento TDA não influenciou na dinâmica da vibração horizontal no posto de operação.

A partir da análise dos dados é possível identificar, de modo geral, que o trator equipado com pneu 13.6 apresentou maiores amplitudes para aceleração vertical e longitudinal estão situadas entre 4 e $6 \mathrm{~Hz}$, respectivamente (Figuras 4 e 5). Nesta frequência, trabalhando com o Transformax ${ }^{\circledR}$, na faixa de $5 \mathrm{kN}$ e $14,33 \mathrm{rpm}$, o operador pode chegar a uma jornada de trabalho diária de 16 horas, sem que ocorra danos a sua saúde segundo a ISO 2631 (1997). Passando da menor força de tração e rotação do eixo motriz para faixa de $18 \mathrm{kN}$ 


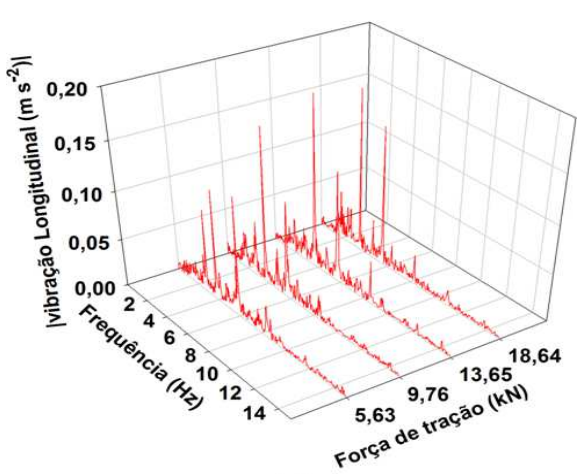

(a)

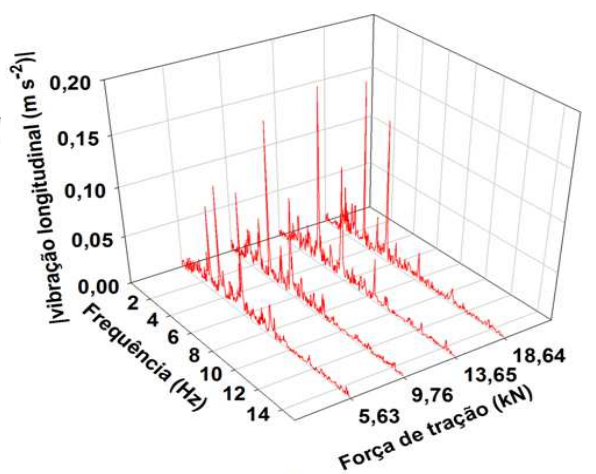

(b)

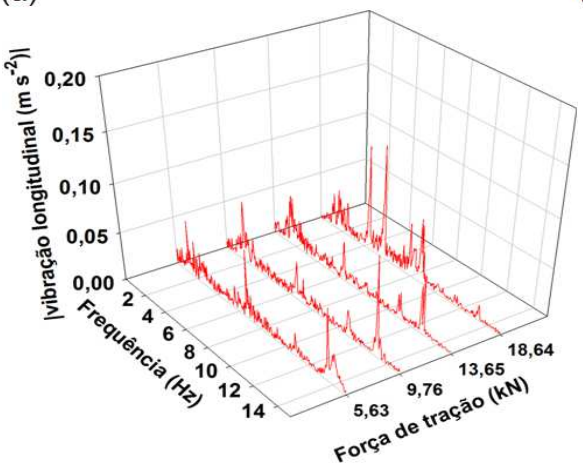

(c)

Figura 4. Espectro de frequências para a vibração Longitudinal na base do posto de operação do trator equipado com pneu 13.6 de acordo com a força de tração testada, nas rotações do eixo motriz1(a), 2 (b) e 3(c).

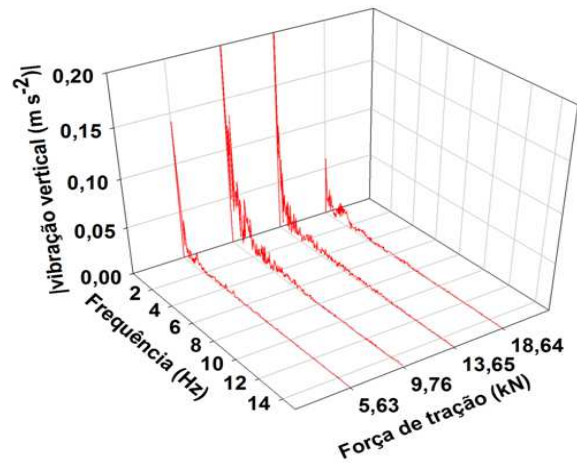

(a)

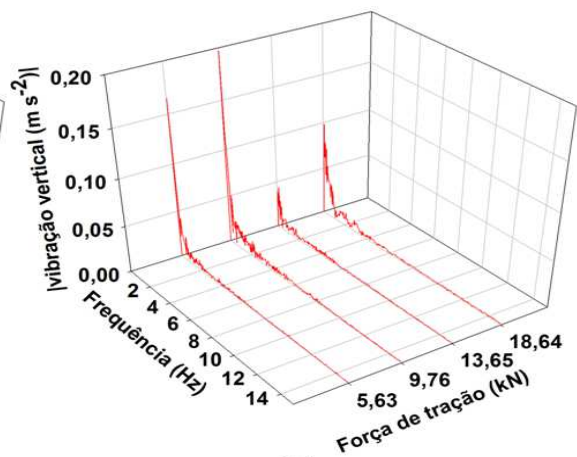

(b)

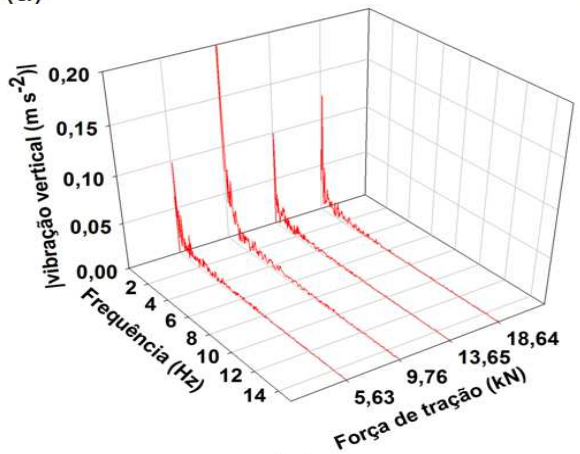

(c)

Figura 5. Espectro de frequências para a vibração vertical na base do posto de operação do trator equipado com pneu 13.6 de acordo com a força de tração testada, nas rotações do eixo motriz1(a), 2 (b) e $3(\mathrm{c})$. 


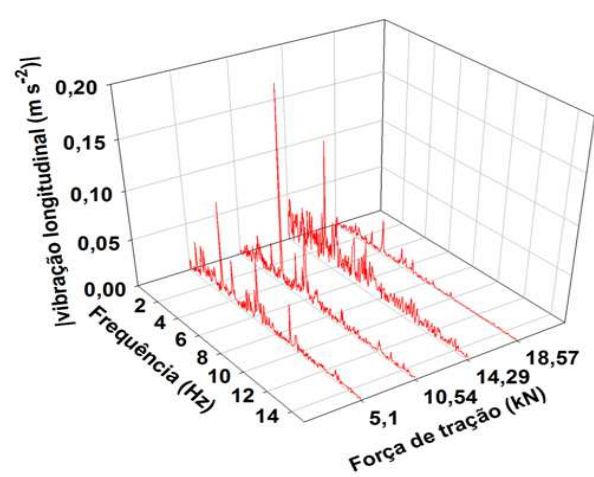

(a)

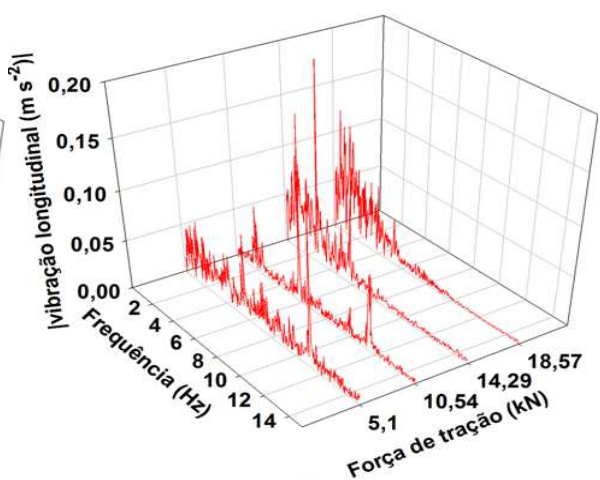

(b)

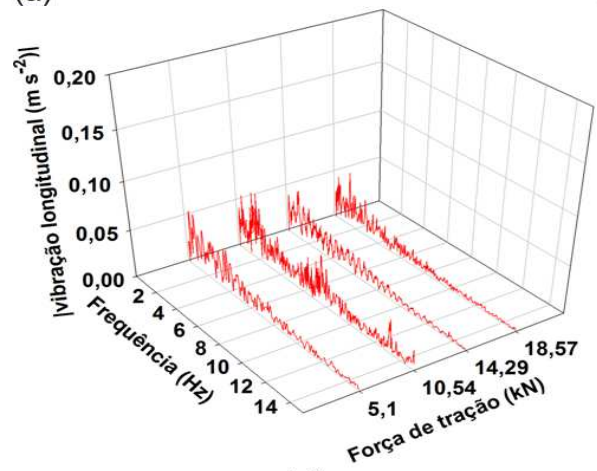

(c)

Figura 6. Espectro de frequências para a vibração longitudinal na base do posto de operação do trator equipado com pneu 18.4 de acordo com a força de tração testada, nas rotações do eixo motriz1(a), 2 (b) e 3(c).

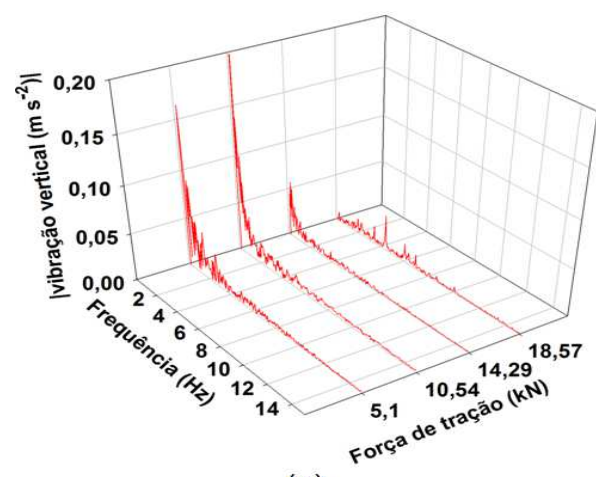

(a)

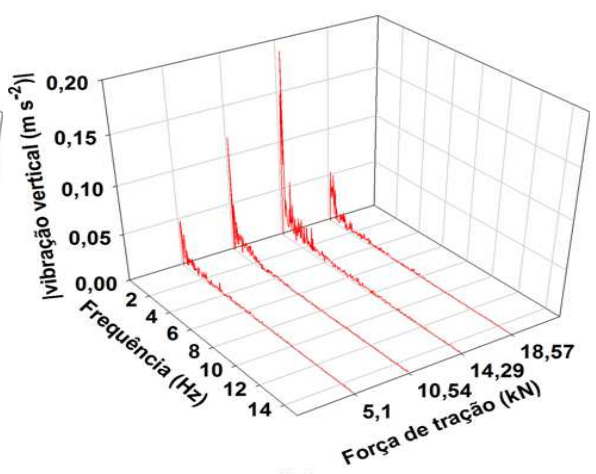

(b)

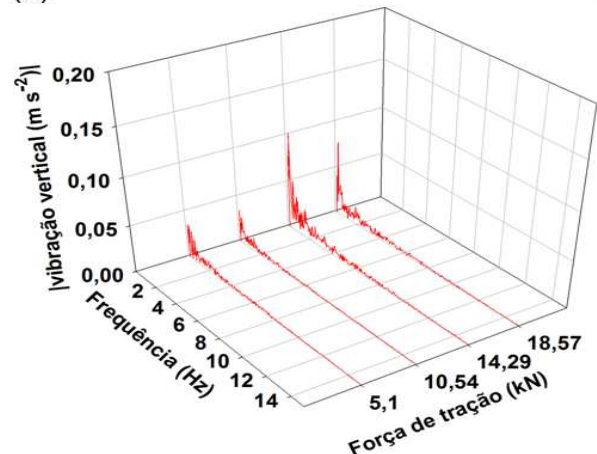

(c)

Figura 7. Espectro de frequências para a vibração vertical na base do posto de operação do trator equipado com pneu 18.4 de acordo com a força de tração testada, nas rotações do eixo motriz 1 (a), 2 (b) e 3(c).

\section{REVENG}

291-301p. ENGENHARIA NA AGRICULTURA, VIÇOSA - MG, V.24 N.4, JULHO / AGOSTO 2016 
e $32 \mathrm{rpm}$, a jornada de trabalho é reduzida para 4 horas diárias.

De acordo com o exposto é possível identificar, de modo geral, que as maiores amplitudes de vibração longitudinal e vertical do trator equipado com pneu 18.4 estão situadas entre de 4 a $2 \mathrm{~Hz}$, respectivamente, (Figuras 6 e 7). Nesta faixa, trabalhando com o trator avaliado tracionando uma força de $5 \mathrm{kN}$ e deslocando-se na rotação do eixo motriz de 14,33 rpm, a jornada de trabalho do operador pode chegar a 24 horas diárias segundo a ISO 2631 (1997), entretanto na maior força e rotação avaliada a jornada de trabalho é reduzida para 8 horas diárias.

Os resultados corroboram com Santos Filho et al. (2003), onde os maiores picos de vibração vertical no assento do operador, para a operação de gradagem, foram encontrados na faixa entre 2 e 4 $\mathrm{Hz}$, dada pela densidade espectral de potência para as três marchas avaliadas.

De acordo com pesquisas, pernas dobradas a $90^{\circ}$ tem uma resposta dinâmica na frequência de $2 \mathrm{~Hz}$, o que pode ser considerado a parte do corpo humano que estaria sujeita a maiores lesões quando combinado com velocidade operacional e uma pressão de insuflação de 12 psi (82,74 kPa) (BRUEL \& KJAER, 2002; VILLIBOR et al., 2015).

$\mathrm{Da}$ mesma forma, estudos experimentais investigaram o desconforto que surge a partir da vibração vertical em todo o corpo sentado em assento rígido, sem encosto e com encosto, com $8 \mathrm{~Hz}$ ou acima deste valor de vibração vertical, ocorreu situação de desconforto, enquanto que em frequências em torno de 5 e $6,3 \mathrm{~Hz}$ houve menos desconforto quando se utiliza um encosto inclinado (BASRI e GRIFFIN, 2013).
As variáveis rotação do eixo motriz e força de tração não apresentaram diferença significativa ao nível de $5 \%$ de probabilidade pelo teste $t$, nos níveis de ruído no posto de operação do trator estudado quando equipado com ambos os pneus avaliados (Quadro 2).

Mantendo sempre o mesmo regime de rotação de trabalho do motor (2100 rpm) durante os testes, os valores médios do nível de ruído no posto de operação do trator avaliado foi de 81,8 e $83,5 \mathrm{~dB}$ para o pneu 13.6 e 18.4 , respectivamente, valores abaixo do limite de $85 \mathrm{~dB}$, para uma jornada de 8 horas de trabalho sem protetor auricular, segundo a NR-15 (MTE 2008). Este parâmetro mostrou que a cabine da máquina estudada apresentou uma eficiência na vedação contra ruídos externos, produzidos pelo motor da máquina, liberação dos gases da exaustão do motor.

Entretanto o nível de ruído mais elevado no posto de operação do trator avaliado equipado com pneu 18.4. Este comportamento pode ser justificado pelo fato do dispositivo de tração contribuir para maior potência na barra de tração, fator que demandou maior torque do motor exigindo maior consumo de combustível, consequentemente, aumentando o fluxo de gases proveniente da combustão, que ao passarem pelo dispositivo de liberação dos gases (escape) elevou o nível médio de ruído no posto de operação.

Ao estudarem a influência da velocidade de trabalho e a condição do solo nas operações agrícolas na determinação dos níveis de potência sonora (ruído) emitido pelo trator agrícola de $55,2 \mathrm{~kW}$ de potência no motor, Arcoverde et al. (2011) verificaram que os valores mais relevantes

Quadro 2. Equações de regressão ajustadas para o ruído (Rpo em $\mathrm{dB}$ ) do trator em função da rotação do eixo e da força de tração, o respectivo coeficiente de determinação $\left(\mathrm{R}^{2}\right)$ e a média do ruído durante o experimento para cada um dos pneus avaliados.

\begin{tabular}{lccc}
\hline Pneu & Equações ajustadas & R2 & $\begin{array}{c}\text { Média do Ruído } \\
(\mathbf{d B})\end{array}$ \\
\hline 13.6 & $\mathrm{Rpo}=81,4247-0,0611^{\text {n.s. }} \mathrm{F}+0,0549^{\text {n.s. }} \mathrm{R}$ & 0,2384 & 81,89 \\
\hline 18.4 & $\mathrm{Rpo}=81,1526+0,1280^{\text {n.s. }} \mathrm{F}+0,0386^{\text {n.s. }} \mathrm{R}$ & 0,5572 & 83,51 \\
\hline $\begin{array}{l}\text { n.s. }- \text { Significativo ao nível de } 5 \% \text { pelo teste t. Rpo }- \text { Ruído no posto de operação }(\mathrm{dB}), \mathrm{R}-\text { Rotação do eixo motriz }(\mathrm{rpm}) \mathrm{e} \mathrm{F}-\mathrm{Força} \\
\text { de tração }(\mathrm{kN}) .\end{array}$
\end{tabular}


foram encontrados no conjunto trator e grade, que apresentou elevada potência na barra de tração exigindo maior potência do motor, o que por sua vez promoveu o aumento no nível de ruído no posto de trabalho.

$\mathrm{Na}$ avaliação do nível do ruído no posto de trabalho de dois tratores de $55,2 \mathrm{~kW}$ (sem cabine) e 80,9 kW (cabinado) em função da velocidade de deslocamento, pressão interna dos pneus e tipo de pista, Tosin et al. (2009) encontraram o nível de pressão sonora menor no trator de $80,9 \mathrm{~kW}$, embora este tenha uma potência maior que o de $55,2 \mathrm{~kW}$, segundo os autores isto se deve ao fato do trator ser cabinado, o que funciona como uma superfície refletora do som. Franklin et al. (2006), na avaliação de ruído no posto de operação de tratores com e sem cabine, mostraram que as cabines nos tratores podem reduzir o nível de ruído em $16 \mathrm{~dB}$.

\section{CONCLUSÕES}

- A força de tração e a rotação do eixo motriz não apresentaram efeito significativo nos níveis de ruído no posto de operação do Transformax ${ }^{\circledR}$.

- O acréscimo da aceleração vertical e longitudinal no posto de operação do trator equipado com pneu 13.6 e 18.4 ocorreu em função do aumento da força de tração e rotação do eixo motriz.

\section{REFERÊNCIAS BIBLIOGRÁFICAS}

ALVES, A.D.S.; COSTA, F.R.L.; CORTEZ, J.W.; DANTAS, A.C.S.; NAGAHAMA, H.J. Nível de potência sonora emitidos por trator agrícola em condições estáticas e dinâmicas. Pesquisa Agropecuária Tropical, v.41, n.1, p.110-119, 2011.

ARCOVERDE, S.N.S.; CORTEZ, J.W.; PITANGA JUNIOR, C.O.; NAGAHAMA, H.J. Nível de potência sonora emitido nas operações agrícolas. Revista Nucleus, v.8, n.1, p.277-287,2011.

BASRI, B.; GRIFFIN, M.J. Predicting discomfort from whole-body vertical vibration when sitting with an inclined backrest. Applied Ergonomics, v.44, n.3, p.423-34, 2013.
BOUAZARA, M.; RICHARD, M.J.; RAKHEJA, S. Safety and comfort analysis of a 3-D vehicle model with optimal non-linear active seat suspension. Journal of Terramechanics, v.43, p.97-118, 2006.

BRASIL. Ministério do Trabalho e Emprego. Atividade e operações insalubres. NR15. Disponível em: <http://portal.mte.gov.br/ legislacao/norma-regulamentadorra-n-15-1.htm>. Acesso em: 10 de jan 2015.

BRÜEL e KJAER, Human Vibration, Brüel e Kjaer Sound and Vibration Measurement: Technical Presentation, Skodsborgvej, 2002.

CUNHA, J.P. Avaliação dos níveis de vibração e ruído emitidos por um trator agrícola em preparo do solo. Revista Pesquisa Agropecuária, v.39, n. 4, p.348-355, 2009.

DHINGRA, H.S.; TEWARI, V.K.; SINGH, S. Discomfort, pressure distribution and safety in operator's seat - A critical review. Agricultural Engineering International: The CIGR Journal of Scientific Research and Development, v.5, p.116, 2003.

ISO 2631-1. "Mechanical vibration and shock - evaluation of human exposure to wholebody vibration - Part I: general requirements". Switzerland: International Standard, 1997.

FERNANDES, H.C.; BRITO, A.B.; MINETTI, L.J.; SANTOS, N.T.; RINALDI, P.C.N. Avaliação ergonômica da cabine de um trator florestal. Revista Ceres, v.57, n.3, p.307-314, 2010.

FIEDLER, N.C.; GUIMARÃES, P.P.; ALVES, R.T.; WANDERLEY, F.B.; Avaliação ergonômica do ambiente trabalho em marcenarias do sul do Espírito Santo. Revista Árvore, v.34, p.907-915, 2010.

FRANKLIN, R.C.; DEPCZYNSKI, J.; CHALLINOR, K.; WILLIAMS, W.; FRAGAR, L.J. Factors affecting farm noise during common agricultural activities. Journal of Agricultural 
Safety and Health, v.12, n.2, p.117-125, 2006.

KILESSE, R.; FERNANDES, H.; SOUZA, A.; MINETTE, L.; TEXEIRA, M.M. Avaliação de fatores ergônomicos em postos de trabalho de motoristas de caminhões utilizados no meio agrícola. Engenharia na Agricultura, v.14, n.3, p.202-211, 2006.

KROEMER, K.H.E., GRANDJEAN, E. Manual de Ergonomia: Adaptando o trabalho ao homem, $5^{\circ}$ edição Artmed Editora, Porto Alegre, 2005.

LANGER,T.H.;EBBESEN,M.K.;KORDESTANI, A. Experimental analysis of occupational wholebody vibration exposure of agricultural tractor with large square bale. International Journal of Industrial Ergonomics, v.47, p. 79-83,2015.

LIDA da I. Ergonomia: projeto e produção. $9^{a}$ ed. São Paulo, Edgard Blücher. 465p, 2003.

LIMA-JUNIOR, P.S.; CORTEZ, J.W.; NAGAHAMA, H.J.; ARCOVERDE, S.N.S. Ruído em função da rotação, raio de afastamento, velocidade de trabalho e tipo de pista de um conjunto trator-carreta cafeeira. Revista Agraria, v.7, n.26, p.581-582, 2014.

LOUTRIDIS, S.; GIALAMAS, T. GRAVALOS, I.; MOSHOU, D. KATERIS, D. XYRADAKIS, P.; TSIROPOULOS, Z. A study on the effect of electronic engine speed regulator on agricultural tractor ride vibration behavior. Journal of Terramechanics, v.48, p.139-147, 2011.

MARSILI, A.; RAGNI, L.; SANTORO, G.; SERVADIO, P.; VASSALINI, G. Innovative systems to reduce vibrations on agricultural tractors: comparative analysis of acceleration transmitted through the driving seat. Biosystems Engineering, v.81, p.35-47, 2002.

MEHTA, C.; SHYAM, H.; SINGH, P.; VERMA,
R. Ride vibration on tractor-implement system. Applied Ergonomics, v.31, p.323-328, 2000.

\section{MINISTÉRIO DO TRABALHO E DO EMPREGO} (MTE). Atividades e operações insalubres: NR15. 2008. Disponível em: <http://www.mtb.gov.br/ legislacao/normas_regulamentadoras/nr_15.asp $>$. Acesso em: 5 out. 2015.

PINHO, M.S.; SCHLOSSER, J.F.; FRANTZ, U.G.; FARIAS, M.S.; UHRY, D.; RIBAS, R.L. Acelerações eficazes na interface assento-operador de um trator. Ciência Rural, v.44, n.10, p.17971803, 2014.

RIBAS, R.L.; SHLOSSER, J.F.; FRANTZ, U.G.; FARIAS, M.S.; RODRIGUES, F.A. Exposição humana à vibrações de corpo inteiro em um trator agrícola com pneus radiais. Ciência Rural, v.44, n.9, p.1589-1595, 2014.

SANTOS FILHO, P.F.; FERNADES, H.C.; QUEIROZ, D.M.; SOUZA, A.P.; CAMILO, A.J. Avaliação dos níveis de vibração vertical no assento de um trator agrícola de pneus utilizando um sistema de aquisição automática de dados. Revista Árvore, v.27, p.887-895, 2003.

SARLETT, A.J., PRINCE, J.S., STAYNER, R.M. Whole-body vibration: Evaluation of emission and exposure levels arising from agricultural tractors. Journal of Terramechanics, v.44, n.1, p. 65-73, 2007.

TOSIN, R.C.; LANÇAS, K.P. ARAUJO, J.A.B. Avaliação do ruído no posto de trabalho em dois tratores agrícolas. Revista Energia na Agricultura, vol.24, n.4, p.108-118, 2009.

VILLIBOR, G.P.; SANTOS, F.L.; FURTADO JÚNIOR, M.R. Mechanical vibrations at the seat base of a tractor during the subsoiling operation. Revista Agrarian, v.8, n.27, p.64-73, 2015. 